\title{
The Contribution of the Internet into Learning
}

\author{
Efstratios Papanis \\ Assistant Professor, Department of Sociology, University of the Aegean \\ Theofilou Hatzimichael 5, 81100 Mytilene-Lesvos, Greece \\ Tel: 30-22510-36520Ｅ-mail: papanis@papanis.com \\ Panagiotis Giavrimis \\ Lecturer, Department of Sociology, University of the Aegean \\ Pamfilis 17, 81100 Mytilene-Lesvos, Greece \\ Tel: 30-22510-36554Ｅ-mail: giavrimis@soc.aegean.gr \\ Eirini-Myrsini Papani \\ Med, Phd Student, Department of Sociology, University of the Aegean \\ Parou 11, 81100 Mytilene-Lesvos, Greece \\ Tel: 30-22511-00283Ｅ-mail: myrena@gmail.com
}

\begin{abstract}
Nowadays, the expansion of the Internet is, undoubtedly, widespread and has developed a new socio-economic environment, where information, innovation and knowledge play a primary role. Through its multiplicity the Internet constitutes probably the best way for accessing entertainment, learning and information, as well as for establishing socialization processes and communication among people.

This paper examines issues related to the learning process, the learning environments developed by the new virtual reality and the relationship between learning and the Internet, with a particular focus on the impact of the Internet on informal learning processes. The survey mainly aims at investigating university students' beliefs about the impact of the Internet on the learning process. The sample is comprised by 390 students from various Greek university departments, 160 (41\%) males and 230 (59\%) females.

The majority of the students believe that the Internet can significantly contribute into the learning process. More specifically, they state that the Internet use can improve learners' academic performance, promote research skills and critical thinking, encourage independent or collaborative learning, enhance motivation, strengthen self-confidence and improve the teaching methods. It facilitates the access to information that the educational system fails to provide, and offers knowledge, frequently more useful than that provided by the courses, complementing, thus, "formal" learning. The research findings also show a differentiation in Internet use, which is associated with the educational level of students' parents.
\end{abstract}

Keywords: Internet, Informal learning, University students

\section{Introduction}

For centuries various philosophical, sociological and psychological currents argue that the interpretation of phenomena cannot be independent by the person who observes them. In other words, the observer affects and is affected by the social reality he/she studies. The implications of this concept - prevalent in the thinking of the presocratic philosophers, strengthened by the phenomenology and supported by the critical philosophy - can lead to the ultimate relativism or cause the heated reactions of those supporting the empirical theory and methodology. However, it is nowadays that their implementation is fostered more than ever before, since the analysis of virtual networks and the examination of the relations developed through computer-based communication, make their application necessary.

Although for the uninitiated or for a huge portion of the population without access to Internet all the above seem meaningless and unnecessary, however, cyberspace and its symbolisms constitute the best reflection of the 
emergence and development of a dynamic social reality, which presupposes research flexibility for its study and interpretation.

The relations, the culture, the stereotypes and the networks developed in the cyberspace and the importance given to them are genuine and authentic, even if they do not take place in face-to-face, real world interactions. Thus, the restrictions of this "keyboard communication" and the possibility for the concealment of the real identity of the users do not prevent - according to social constructivism - the development of real social experiences and relations.

The term "virtual communities" was created to describe the relations, the multiplicity of roles, the culture and the philosophy, the language, the rules, the ethics and the norms developed in the Internet, which are beginning gradually to affect the mentality of the community members and the whole society, in direct and indirect ways. Through the study of virtual communities one can better understand the daily social experience and make steps towards self-awareness. Communication on the Internet has so many specificities, that we can now talk about a different dimension of it, based on quantitative and qualitative criteria.

Despite the complexity of the multimedia environment of a game, an internet community, a group discussion or chat and despite the complexity of the graphics, what is being drastically changed is the non-verbal communication, the language of the body. Emphasis is mainly given on written discourse and the cognitive functions of encoding and decoding it entails. The walk with a friend, the facial expressions, the movements of the body, the smell, the touch and the taste lose the important role they play in real life. Thus, the Internet users may opt to present only selected information about themselves, to remain anonymous, to introduce their real identity and personality or to create a new self, according to their self-esteem and intentions. In games like "Second Life", "There", and "Palace", which are expected to replace the frenzy of Facebook, a player dresses up a virtual costume, a virtual reflection of him/herself (avatar) and lives a second, parallel life, seizing the opportunity to display the most hidden instincts and desires, to demonstrate an ideal self, or to be "sunk" into a symbolic, virtual reality, which relieves the stress and the stereotypes of everyday life. When people feel that the consequences of their actions will have no impact on real life and that will not threaten their real self, easily remove the social conventions and are released from the psychological mechanisms imposed by the superego. The attitudes which are developed in the Internet and are defined by anonymity do not really "belong" to the Internet user and may not affect the sense of self. The distance from the real identity exonerates the user and helps him/her rehearse aspects of the personality that otherwise would remain trapped in the subconscious. Communication through the Internet releases from conventions: while writing an e-mail there is no need to be decently dressed, and the body language, which would intercept or facilitate communication, is not available to the receiver of the message. Murray (1991) found that the language used on the Internet is governed by its own cognitive and psychological mechanisms. Moreover, Shank (1993) concluded that this language should be characterized as semantic, instead of written or oral. Internet communication may refer to two users, to one who communicates with many others or with a particular group, or to many users.

The addiction resulting from this situation is obvious. The user is in an hypnotic situation, where consciousness is seriously affected: in the safety of a room, in an environment without the risks of the painful and stressful life, the user follows his/her own pace, has a different sense of cause and effect and obtains enhancing virtual experiences in the democratic environment of the Internet, which emerges as opposed to the plethora of obligations of daily life: the user is free to decide his/her gender and age, and select the websites, where navigation and communication will take place. The social status, the educational level and personal achievements are not being evaluated; what is important is the degree of familiarity with the computer technology, the games, the chats and discussions, the forums and the groups in which the user wants to be integrated. Anyone can become an active Internet user, making his/her presence known to other users and demonstrating ideas and opinions. Of course, statistics on Internet use show that poor countries and population groups like homeless people and the socially excluded, do not participate in the world of the Internet.

While on the Internet, the sense of time is dramatically altered. Plenty of opportunities for synchronous and asynchronous communication are provided. The electronic mail and the participation in newsgroups permit the user to respond in his/her own pace. Moreover, even while chatting, there is much more time for response, in comparison to real life. This creates ideal conditions for reflection and storage/recording of the conversations, in order to draw secure conclusions and develop appropriate communicative strategies. The Internet is evolving so quickly, that the participation of some months in a game or a group is enough for the user in order to be characterized as an experienced member of the virtual community. 
Finally, the language ceases to be subject to physical constraints, is decorated with emoticons, enriched with neologisms, abbreviations and mixed languages and ultimately determined, not by grammatical and syntactical rules, but by the way the user internalizes the world.

\section{Internet and Informal Learning}

In the post-industrial era the various cognitive tools and particularly the Information and Communication Technologies (ICT) enhance all types of learning. One of the most important ICT applications, which has an impact on the way people interact, is the Internet. It constitutes an active and creative learning environment and an important cognitive tool, which contributes to the effectiveness of the teaching and the learning process (Charp, 1998).

According to research done (Catledge \& Pitkow, 1995) about the reasons people navigate the Internet, some Internet users just browse, some discover information by chance, and others look systematically for specific information. Each of these reasons represents a specific type of learning (Calvani \& Rotta, 1999), as the person starts to look for general information and later focuses on specific information. In each case, the person obtains knowledge that is either independent or part of a cognitive schema.

The Internet provides a type of Informal learning. It is based on communication tools, such as the electronic-mail, the chat rooms, the forums and tools for accessing the information, such as browsers, websites and data bases. The communicative effectiveness of information sources, the possibilities for navigation and searching of information, the observation and discussion with no time limits, explain why the Internet can have special teaching functions and qualities. It combines technology with a plethora and variety of sources, virtual experiences, work and leisure, social relations, which encourage the learners to be more creative and flexible (Heo et al., 2007; Livingstone, 2003).

Through the Internet there is access to a virtual reality, which can substantially alter the way learning is achieved. Learners are not passive receivers of information, but active explorers of it. They are successfully involved in the learning process, since the virtual reality requires the use of students' cognitive, social, intellectual and emotional abilities (Raptis \& Rapti, 2002). The Internet, therefore, through simulated environments, virtual worlds, collaborative networking environments and experiential groups, permits the combination of a variety of learning methods, such as the systematic, the independent, the collaborative, the lifelong, the distance, and the open, online learning.

The complexity and the structure of the Internet significantly expand the boundaries of learning. Through visualization, data and concepts are clearly represented, a fact that facilitates the comprehension and the assimilation of information (Ala-Mutka \& Kirsti, 2009; Mitra \& Steffensmeier, 2000). Being a constantly evolving informational environment, it constitutes a learning experience and contributes to the development of different learning types and to the satisfaction of various learning styles, either through information searching or through simple navigation (Passerini \& Granger, 2000). Thus, various types of learning are accomplished; learning through simulation and the experience of the virtual reality, through exploration, reflection and case studies, as well as accidental learning.

Obviously, the Internet promotes individual learning, since each user, according to the personal objectives set, is free to conduct researches and explorations in an environment consisting of a huge amount of information. This information is accessed quickly and easily and the user can experiment and exploit the numerous possibilities offered gaining virtual experiences (Calvani. \& Rotta, 1999). Thanks to this exploration of the web space accidental learning is accomplished. The Internet is supportive to the achievement of the learning objectives, since, through the use of appropriate media (supernatants, hypermedia) transmits knowledge and information and enhances people's interaction (Muffoletto, 1994; Raptis \& Rapti, 2002). The increasing use of the Internet and of the communication tools available, enhances informal learning. The number of the people who gain access to knowledge and information is rapidly increasing, while the search engines and the forms of interaction are also multiplied.

\section{The research aim}

In the light of the above, the present research aims at recording students' opinions about the impact of the Internet on education, at studying the various ways in which it affects informal learning and at identifying the aspects of education which are based on the Internet use. 


\section{Methodology}

\subsection{The sample}

The sample of the research consists of 390 students - 160 males (41\%) and 230 females (59\%) - studying in various Greek university departments. Most of the respondents are in the $3^{\text {rd }}(24.9 \%)$ and in the $4^{\text {th }}(28.2 \%)$ year of studies. The $18.4 \%$ of the sample is comprised by students who are in the $5^{\text {th }}$, the $6^{\text {th }}$ and the $7^{\text {th }}$ year of studies, the $17.7 \%$ attends the $2^{\text {nd }}$ year, while only the $10.5 \%$ is in the first year. The majority of the parents have a degree in secondary education (the $43.1 \%$ of the fathers and the $43.8 \%$ of the mothers). High is also the percentage of the parents who possess university degrees (the $32.6 \%$ of the fathers and the $26.9 \%$ of the mothers). On the contrary, the $8.7 \%$ of the fathers and the $10.5 \%$ of the mothers possess only a primary education diploma.

\subsection{The research instrument}

The research instrument is an improvised questionnaire exploring the use of new technologies and the Internet, as well as their effects on the users. Its construction was based on international and Greek literature concerning the theoretical models for learning in general and for informal learning in particular, as well as on research done on the effects of the Internet. The first part of the questionnaire aims at eliciting basic socio-demographic information, such as the gender, the year of studies and parents' educational level. The next part includes questions which concern computer and internet accessibility, while the third part contains questions about the Internet use and the fourth questions on the socio - affective and the cognitive effects of this use on the individuals. Finally, the questions of the last part aim at the evaluation of the Greek educational system. Frequency analysis and non-parametric methods for data processing were used in the present research.

\section{Results}

\subsection{Frequency analysis}

A fairly high percentage of students, approximately the $73.8 \%$, has Internet access at home. Furthermore, the $81.3 \%$ of the sample states that can easily access a computer at the university, while the $18.7 \%$ claims the opposite. This is also supported by the fact that the $91.8 \%$ has an easy access to the Internet at the university, while only the $8.2 \%$ has no access. Moreover, the $72.6 \%$ of the asked population can access a computer at other places, apart from home and the university, while a significant percentage of around $27.4 \%$ cannot.

Regarding the frequency of connection to the Internet, the largest percentage (64.9\%) states that is connected daily, the $21.5 \%$ only a few times a week, the $8.5 \%$ sometimes a month and the $5.1 \%$ less than once a month. The $73.6 \%$ of the sample knows how to get information from a database, while the $26.4 \%$ does not. The $46.7 \%$ of the students sometimes makes use of educational software, while approximately the $27.9 \%$ never does.

More than the $50 \%$ of the students believe that the Internet has a special learning role as they state that a) it helps to improve their academic performance, b) promotes research skills, c) increases their motivation for learning, c) permits and facilitates independent learning, d) differentiates them cognitively, e) enhances users' critical thinking and self-esteem, f) enriches the teaching methods and g) satisfies learners' needs and interests more effectively and essentially than the existed educational system.

However, there are university students, whose percentage is ranging between $13 \%$ and $38 \%$, who state that in some cases they probably do not agree or disagree with these views. It is noteworthy that the students have not a clear opinion about the impact of the Internet on the role of the teacher, and that most of them believe that it cannot improve the attention and the concentration of the learner.

\section{Table 1 about here}

As far as the effects of the Internet on learners' socio-effective skills are concerned, the students stated that through Internet use learners' cooperation is increased, their positive interaction is enhanced and new interpersonal relationships are developed and essentially facilitated. Moreover, the Internet use seems to have a positive impact on the strengthening of confidence and self-esteem.

\section{Table 2 about here}

Compared with the existed educational system, most respondents said that a) the Internet helps to acquire knowledge that the educational system is unable to provide, b) provides information that is more useful than that offered by the courses, c) the information reached through Internet navigation does not contradict what they are taught -the $27.2 \%$ of the respondents, however, believes that this contradiction is sometimes identified, while the $5.9 \%$ claims that it always occurs- and c) supplements knowledge on issues of interest. However, a proportion, ranging between $8.5 \%$ and $43 \%$, disagrees with the above stated and argues that the Internet does not effectively contribute to knowledge acquisition and that the information provided is not really useful. 


\section{Table 3 about here}

The analysis Kruskal - Wallis was used to investigate whether there is a statistically significant difference between the answers of the respondents and the educational level of their parents. As far as the educational level of the father is concerned, there is a statistically significant difference in the following questions: "The Internet increases and facilitates cooperation with other persons" ( $\mathrm{x}^{2}=9,860 \mathrm{df}=3$ and sig $=0,020<0,05$, Mean Rank Primary School=244,51, Mean Rank High School=208,07, Mean Rank Senior High school=184,4, Mean Rank University/Technological Institute= 191,02), "Enhances learners' positive interaction" $\left(\mathrm{x}^{2}=23,234 \mathrm{df}=3 \mathrm{and}\right.$ sig= 0,000<0,05, Mean Rank Primary School= 230,06, Mean Rank High School= 247,96, Mean Rank Senior High School= 179,71, Mean Rank University/Technological Institute= 181,94). In the rest of the questions no statistically significant difference was identified. It is concluded that the students with fathers of low educational level (Primary School, High school) are more affected than those whose fathers are highly educated.

As far as the educational level of the mother is concerned, there is a statistically significant difference in the following questions: "The Internet differentiates learners cognitively" ( $\mathrm{x}^{2}=12,732, \mathrm{df}=3$ and $\mathrm{sig}=0,005<0,05$, Mean Rank Primary School= 150,1, Mean Rank High School=181,49, Mean Rank Senior High School= 211,99, Mean Rank University/Technological Institute $=194,42)$, "Changes the role of the teacher" $\left(\mathrm{x}^{2}=7,841, \mathrm{df}=3\right.$ and sig $=0,049<0,05$, Mean Rank Primary School = 208,62, Mean Rank High School= 223,83, Mean Rank Senior High School= 188,44, Mean Rank University/Technological Institute = 182,18), "Offers information more useful than that provided by the educational system" $\left(\mathrm{x}^{2}=13,371, \mathrm{df}=3\right.$ and $\mathrm{sig}=0,004<0,05$, Mean Rank Primary School= 241,07, Mean Rank High School= 200,12, Mean Rank Senior High School= 197,94, Mean Rank University/Technological Institute $=170,51)$. It is concluded that the students with mothers of low educational level (Primary School, High school) are more affected than those whose mothers are highly educated. In the rest of the questions no statistically significant difference was identified.

\section{Discussion}

The rapid and continuous development of sciences and technologies and the strong socio-economic transformations are significantly influencing both the social structure and the organization of the educational systems. The Internet as a cognitive tool has created a new socio-economic environment, where information, innovation and knowledge have a leading role. It is probably the best and the easiest way for accessing entertainment and information and for facilitating learning and socialization. It has been linked to multiple variables such as the gender, the socio-economic status of the individuals, the structure and function of the society, the family and especially the educational systems.

The question which often arises as a social and educational issue is whether and to what degree the Internet actively contributes to knowledge acquisition and to learning facilitation. There are many theorists who support that it constitutes an effective cognitive tool, as it enables the individual to look for information, to exchange it, to cooperate and discuss, acquiring, thus, a more complete and holistic view of an issue. On the other hand, there are also theorists who question the learning role and the quality of the Internet.

According to the results of the present research, the majority of the students can access the Internet and makes daily, heavy use of it. Students are helped to regulate, organize and direct their learning and to improve their academic performance. Moreover, through Internet use their active and autonomous behavior is enhanced, and the free self-expression is encouraged, a fact that promotes and facilitates collaborative learning and the teaching methods. Furthermore, the Internet offers opportunities for the interdisciplinary approach of a subject area, permitting multiple analyses and representations. The individuals feel autonomous and free to determine their learning processes, giving mental representations to the digital environment of the Internet and structuring their own cognitive schemata.

The students of the specific research state that through their everyday experience with the Internet people develop various skills and accomplish their learning goals. More specifically, they stress that it enhances skills associated with learning, research, academic performance, interpersonal communication and teaching methods. There is, of course, a number of people who do not agree with the above, which may be due to the nature of their Internet use or to the difficulties they face in accessing a computer and Internet - it should be noted that accessibility is still difficult for a quite large part of the population and that only recently the Greek schools have been equipped with an adequate number of computers. Informal learning accomplished through Internet appears to complement both formal learning and the previous experience of the individual. Students state that the Internet contributes to the acquisition of knowledge that the existed educational system fails to provide and that the knowledge gained is often more useful than that obtained by the courses. However, the majority of them indicate that the information found on the Internet does not contradict what they are taught during their studies. It is, also, 
supported that the Internet improves the teaching methods, since the educational system in general and the teachers in specific adapt and adjust their role in line to the new reality. Nevertheless, the educational system, despite the efforts, has not yet reached the desired level of quality and efficiency.

Noteworthy is the differentiation in students' beliefs about the importance of the effects and the contributions of the Internet, according to their parents' educational level. The higher the educational level of the father and the mother, the smaller the effect of the Internet on learners' cognitive skills and interactions.

The complete comprehension of the functions and the effects of the Internet requires in depth analysis, as it constitutes a complex mechanism for producing knowledge. The evaluation of its quality and effectiveness, particularly in education, is, undoubtedly, a difficult task. However, it is necessary to understand its functions, in order to find the most appropriate ways for its exploitation during the process of learning.

\section{References}

Ala-Mutka, Kirsti. (2009). Learning in Online Spaces and Communities - how, what and when? Presentation at the Learncom expert workshop, 31 March 2009. [Online] Available: http://is.jrc.ec.europa.eu/pages/EAP/documents/Session4.pdf (February 11, 2010).

Calvani, A., \& Rotta, M. (1999). Comunicazione e Apprendimento in Internet. Roma: Centro Studi Erickson.

Catledge, L. D., \& J. E. Pitkow. (1995). Characterizing browsing strategies in the World-Wide Web. Computer Networks and ISDN Systems, 26(6), 1065-1073.

Charp, S. (1998). Preparing the $21^{\text {st }}$ century teacher, Journal Technological Horizons in Education, 26,6 - 8.

Heo, G. M., Lee, R., \& Park, Y. (2007). Learning experiences of adult bloggers as self-regulated learners. Andragogy Today: International Journal of Adult \& Continuing Education, 10(1), 249-279.

Livingstone, S. (2003). Young people and new media. London: Sage.

Mitra, A., \& Steffensmeier, T. (2000). Changes in student attitudes and student computer use in a computer-enriched environment. Journal of Research on Technology in Education, 32(3), 417-433.

Muffoletto, R. (1994). Technology and restructuring education: constructing a context. Educational Technology, 34(2), 24-28.

Murray, D. E. (1991). The composing process for computer conversation. Written Communication, 8(1), 35-55.

Passerini, K., \& Granger, M. J. (2000). A developmental model for distance learning using the Internet. Computers \& Education, 34(1), 1-15.

Raptis, A., \& Rapti, A. (2002). Learning and teaching in the era of Information Society A holistic approach. Athens: Self-publishing.

Shank, G. (1993). Abductive multiloguing the semiotic dynamics of navigating the net. The Arachnet Electronic Journal on Virtual Culture, 1 (1). Anline] Available: http://www.ibiblio.org/pub/academic/communications/papers/ejvc/SHANK.V1N1 (February 11, 2010). 
Table 1. Impact on learning and teaching

\begin{tabular}{|c|c|c|c|c|}
\hline & \multicolumn{4}{|c|}{ Percentage } \\
\hline & I Disagree & $\begin{array}{c}\text { I } \\
\text { probably } \\
\text { Disagree }\end{array}$ & $\begin{array}{l}\text { I probably } \\
\text { Agree }\end{array}$ & I Agree \\
\hline Improves academic performance & 11.8 & 15.1 & 42.1 & 31.0 \\
\hline Promotes research skills & 4.1 & 9.2 & 40.8 & 45.9 \\
\hline Increases motivation for learning & 11.8 & 20.8 & 38.7 & 28.7 \\
\hline Permits and facilitates independent learning & 10.0 & 15.9 & 34.6 & 39.5 \\
\hline Differentiates learners cognitively & 12.6 & 24.9 & 41.5 & 20.8 \\
\hline Enhances critical thinking & 9.0 & 23.1 & 45.9 & 22.1 \\
\hline Improves self-esteem and the feeling of competence & 11.3 & 20.5 & 40.5 & 27.7 \\
\hline Improves and enriches the teaching methods & 12.8 & 17.7 & 39.2 & 30.3 \\
\hline $\begin{array}{l}\text { Satisfies learners' needs and interests more effectively and } \\
\text { essentially than the educational system }\end{array}$ & 13.3 & 27.7 & 31.3 & 27.7 \\
\hline Improves learners' attention and concentration & 24.9 & 33.6 & 27.7 & 13.8 \\
\hline Changes the role of the teacher & 22.1 & 27.4 & 31.0 & 19.5 \\
\hline
\end{tabular}

Table 2. Effects on the socio-effective domain

\begin{tabular}{|l|c|c|c|c|}
\hline \multirow{2}{*}{} & \multicolumn{4}{|c|}{ Percentage } \\
\cline { 2 - 5 } & $\begin{array}{c}\text { I } \\
\text { Disagree }\end{array}$ & $\begin{array}{c}\text { I probably } \\
\text { Disagree }\end{array}$ & I probably Agree & I Agree \\
\hline Enhances learners' positive interaction & 15.4 & 26.9 & 32.1 & 25.6 \\
\hline Facilitates cooperation & 16.9 & 16.4 & 30.0 & 36.7 \\
\hline Strengthens confidence and self-esteem & 28.7 & 21.5 & 25.6 & 24.1 \\
\hline Develops interpersonal relationships & 14.6 & 13.1 & 23.8 & 48.5 \\
\hline
\end{tabular}

Table 3. Effects on the cognitive domain

\begin{tabular}{|l|c|c|c|c|}
\hline \multirow{2}{*}{} & \multicolumn{4}{|c|}{ Percentage } \\
\cline { 2 - 5 } & Not at all & $\begin{array}{c}\text { A } \\
\text { little }\end{array}$ & Sometimes & Very \\
\hline Supplements knowledge on issues of interest & 2.6 & 15.4 & 36.7 & 45.4 \\
\hline $\begin{array}{l}\text { The information found on internet contradicts what I am } \\
\text { taught }\end{array}$ & 33.3 & 33.8 & 27.2 & 5.9 \\
\hline $\begin{array}{l}\text { It helps in the acquisition of knowledge not provided by the } \\
\text { educational system }\end{array}$ & 8.5 & 23.8 & 37.4 & 30.3 \\
\hline $\begin{array}{l}\text { Offers information more useful than that provided by the } \\
\text { educational system }\end{array}$ & 18.2 & 24.9 & 41.8 & 15.1 \\
\hline
\end{tabular}

